\title{
DEFORMATIONAL COMPATIBILITY OF AGGREGATE PHASE FOR TAPERING FLOW OF DENSE LIQUID-SOLID MATERIAL
}

\author{
By Anura NANAYAKKARA*, Kazumasa OZAWA** and Koichi MAEKAWA***
}

\begin{abstract}
The aim of this research is to clarify the deformational compatibility for dense aggregate phase of concrete in tapered portions of pipe lines used for concrete pumping. The deformational compatibility, which describes the relationship between the strain rate of aggregate phase (particle assembly) developing over a reference section and sectional mean flow speed of the particle assembly, is indispensable for self consistent one-dimensional computation of dense liquid-solid flow. Visual test and image processing on model concrete under simplified flowing conditions were carried out for development of the mathematical description for deformational compatibility of aggregate phase, in which the second invariant of aggregate phase strain was adopted as a main parameter associated with stress generation due to particle-to-particle interactions. In relating sectional averaged invariant of strains to the mean flow speed of solid phase, dimensions and shape of the pipe, the authors proposed a simplified compatibility, reduced to onedimensional condition, applicable to dense liquid-solid flow in straight and tapered sections of pipe lines.

Keywords: liquid-solid flow, tapered section, deformational compatibility, concrete pumping, visual test
\end{abstract}

\section{INTRODUCTION}

The concrete pumping has become a popular way of transporting concrete due to its efficiency and reduction in labor cost. The popularity of concrete pumping has stimulated engineers to develop not only concrete pumps and pipe line networks ${ }^{1)}$ but also mix design concept of concrete from a view point of pumpability ${ }^{2)}$.

For evaluation of pumpability of fresh concrete in a pipe line, the authors have adopted the multiphase concept ${ }^{3) .4)}$ which can treat transient segregation and flow resistance simultaneously. Authors discussed the sectional averaged one-dimensional analytical approach ${ }^{5)}$ with self-consistency as shown in Fig. 1. Here, momentum transfer and mass-balancing equations for aggregate assembly and mortar phase are specified according to Newton's law of motion and incompressibility conditions respectively.

Concrete consists of large amount of particles with different dimensions and shapes. Generally, the particle interaction governs the global behavior of concrete flow. The phase-to-phase interaction (force transfer between coarse aggregate and mortar constituent particles) is considered in terms of the drag force or so-called "segregation resistance force" in Fig. 1. The particle-to-particle interaction in each phase is evaluated in terms of the phase-developing stresses, for example, pore pressure is the phase-developing stress of liquid. The authors conducted numerical simulation ${ }^{5)}$ and verified by computational results that the phase-developing stress of aggregate phase and the phase-to-phase drag force are sensitive to overall kinematics of dense liquid-solid flow similar to fresh concrete.

Previous multiphase researches ${ }^{3)}{ }^{4)}$ have been mainly concerned to slurry flow including small amount of

* Member of JSCE, M. Eng., Graduate Student, Dept. of Civil Engineering, University of Tokyo

** Member of JSCE, M. Eng., Assistant Lecturer, Dept. of Civil Engineering, University of Tokyo

*** Member of JSCE, Dr. Eng., Associate Professor, Dept. of Civil Engineering, University of Tokyo 


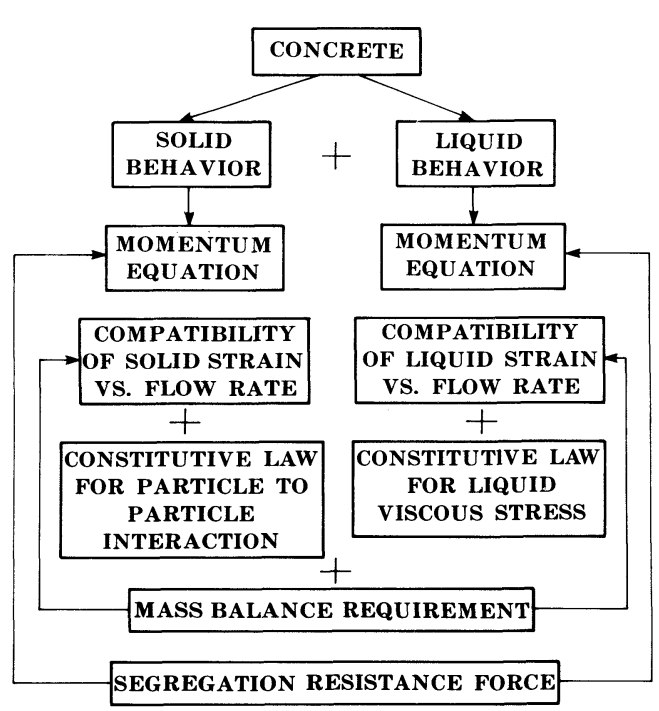

Fig. 1 Two-phase model for fresh concrete.

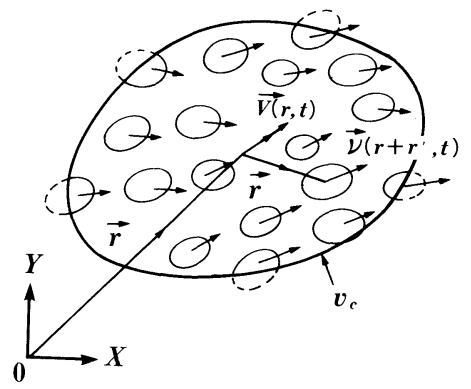

Fig. 2 Definition sketch of position vectors and control volume $v_{c}$
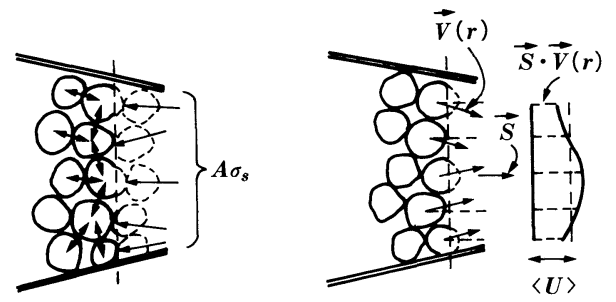

Fig. 3 Aggregate interaction stress $\sigma_{s}$ and sectional averaged flow speed $\langle U\rangle$.

solid particles ( $<30 \%$ by volume) where solid to solid interaction is negligible. So, in this paper, a liquid-solid flow which contains solid of concentration greater than $30 \%$ by volume is referred to as a dense liquid-solid flow.

In order to compute the phase-developing stress of aggregate phase over a flow section, firstly we need the deformational compatibility which gives us the strain rate over a flow section from the flow rate of aggregate, and secondly the constitutive law is needed for computing particle-to-particle stress of aggregate $^{4) .6)}$ as a function of the strain rate in Eulerian expression ${ }^{77}$.

The aim of this paper is to establish the deformational compatibility condition for one-dimensional analysis of pipe flow of dense liquid-solid materials. For this purpose, the deformational mode of aggregate phase related to re-arrangement of aggregate particles must be clarified. But the motion of constituent particles in real concrete cannot be probed just with naked eyes. Then, the visual test method $^{7.8)}$ was adopted to obtain the deformation of aggregate phase in pipe flow of a liquid-solid. A particle processing technique ${ }^{7)}$ based on Eulerian expression was used to clarify dense aggregate phase deformation under various boundary conditions. Special attention has been paid to tapering flow because the strain field in a straight portion of pipe is nearly zero due to plug flow without re-arrangement of particles in case of dense liquid-solid materials.

\section{REDUCED DEFORMATIONAL COMPATIBILITY IN ONE-DIMENSIONAL ANALYSIS}

To obtain the exact expression of particle assembly motion, we need discrete mechanics having degrees of freedom concerned with all processed particles ${ }^{9)}$ 11). This approach is very similar to discrete crack model ${ }^{12)}$ for cracked reinforced concrete mechanics. Otherwise, the multiphase mechanics makes it possible to express the motion of particle assembly continuously with spatially averaged flow vector. The latter approach which the authors adopted is quite similar to the so-called smeared crack model for FE analysis of $\mathrm{RC}^{12}$. Here, we define a spatially averaged flow vectors $\vec{V}$ of aggregate at the location expressed by $\vec{r}(=(x, y, z))$ as 


$$
\vec{V}=\left(u_{x}, u_{y}, u_{z}\right)=\vec{V}(\vec{r}, t)=\frac{\int_{v_{c}} \vec{\nu}\left(\vec{r}+\overrightarrow{r^{\prime}}, t\right) \rho\left(\vec{r}+\overrightarrow{r^{\prime}}, t\right) d v}{\int_{v_{c}} \rho\left(\vec{r}+\overrightarrow{r^{\prime}}, t\right) d v}
$$

where $\overrightarrow{r^{\prime}}$ is incremental position vector defined in the integral volume with the origin denoted by $\vec{r}$ where $\vec{r}^{\prime}$ $=0, \vec{\nu}\left(\vec{r}+\overrightarrow{r^{\prime}}\right)$ is the velocity vector defined on the particles and $\rho\left(\vec{r}+\overrightarrow{r^{\prime}}\right)$ is equal to unity when position vector $\left(\vec{r}+\overrightarrow{r^{\prime}}\right)$ is included in a solid particle, otherwise, $\rho\left(\vec{r}+\overrightarrow{r^{\prime}}\right)$ is zero as shown in Fig. 2 . The integral volume $v_{c}$ is sometimes named as control volume since it includes several particles and the location is denoted by $\vec{r}$ as shown in Fig. 2 .

The general compatibility in three-dimensional flowing field is defined as

$$
\epsilon_{i j}=\frac{1}{2}\left(\frac{\partial u_{i}}{\partial x_{j}}+\frac{\partial u_{j}}{\partial x_{i}}\right)
$$

in which we have strain rates $\epsilon_{i j}$ defined at any location and phase developing stress by using a constitutive relationship. In one-dimensional piping problems, the sectional force dependent on particle-to-particle interaction or sectional averaged stress $\sigma_{s}$ of aggregate phase in the direction of pipe axis is needed. We must be informed with strain rates necessary to calculate stress over a cross section with sectional area $A$ as expressed by Eq. (3) and Fig. 3.

$$
\sigma_{s}=\frac{1}{A} \int_{A} \sigma \cdot\left(\epsilon_{i j}(\vec{r})\right) d A
$$

where function $\sigma$ means the constitutive law for axial stress evaluation due to particle-to-particle interaction and $\vec{r}$ is defined on a pipe section.

In the self consistent system of one-dimensional analysis, however, we have no degree of freedom regarding flow distribution over a cross-section, but have just the sectional averaged flow rate $\langle U\rangle$ in the longitudinal axial direction as defined in Eq. (4) and illustrated in Fig. 3.

$$
\langle U\rangle=\frac{1}{A} \int_{A} \vec{V}(\vec{r}) \cdot \vec{S} d A
$$

where $\vec{S}$ is the unit vector oriented to the axial direction of pipe. Eq. (2) cannot be directly utilized because of reduced degrees of freedom from three-dimension to one-dimension for computing sectional value in Eq. (3).

In classical beam theory we have the same strategy to get continuous one-dimensional mechanics (see Fig. 4). The flexural moment $M$ in beam theory corresponds to sectional averaged stress $\sigma_{s}$ of particles which is needed in the equation of motion (equilibrium). To compute the flexural moment, we need the strain distribution over a section, but the only available information for computing strain is the member deflection $\delta$ at the centroid of the section. Since there is no deformational degree of freedom along a section of the beam, the beam deflection $\delta$ is considered to be corresponding to mean flow rate $\langle U\rangle$ in pipe flow (Eq. (4)).

Here, we have a well-known reduced compatibility based on Euler-Kirchoff hypothesis assuming that the plane section remains plane, that is, normal strain in a section is proportional to the second order differentiation of beam deflection. In other words, this compatibility relates the strain field developing over a section to the characteristic value concerning beam deflection. This is mere empirical formulation with limit of application, but makes it possible to conduct simple one-dimensional beam analysis with reasonable accuracy. In a similar way, deformational compatibility oriented to the sectional stress evaluation is indispensable to establish one-dimensional flow analysis of dense liquid-solid materials including fresh concrete.

The deformation mode or strain rate tensors which are associated with stress generation of aggregate phase must be targeted. In the case of beam theory we refer to a strain component normal to the section only, because other tensorial components have nothing to do with occurrence of flexural moment. Generally, there exist three modes of deformation expressed by strain rate tensors in Eq. (2), that is, 


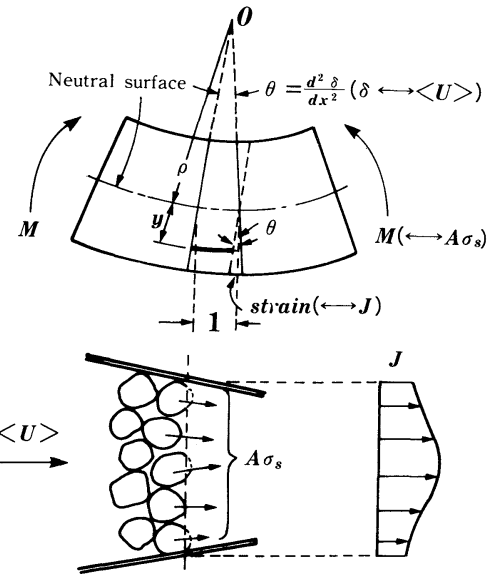

Fig. 4 Deformation of a beam in bending and aggregate phase in tapering pipe flow.

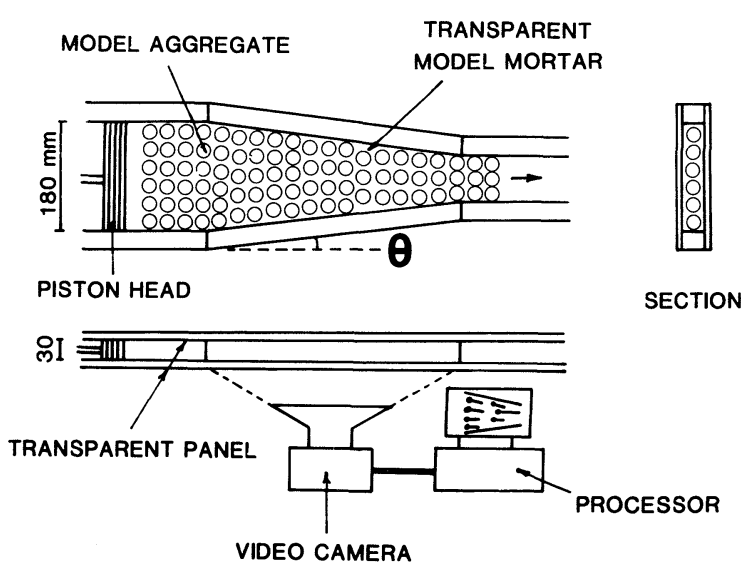

Fig. 5 Pumping apparatus for the visual test.

shear mode denoted by ' $J$ ', volumetric mode denoted by ' $I$ ' and spin mode expressed by $\omega$. In case of two-dimensional particulate kinematics, we have $\mathrm{e}^{\text {() }}$

$$
\begin{aligned}
I & =\frac{\epsilon_{x x}+\epsilon_{y y}}{2} \ldots \ldots \ldots \ldots \ldots \ldots \ldots \ldots \\
J & =\sqrt{\left(\frac{\epsilon_{x x}-\epsilon_{y y}}{2}\right)^{2}+\epsilon_{x y}^{2}} \\
\omega & =\left(\frac{\partial u_{x}}{\partial y}-\frac{\partial u_{y}}{\partial x}\right) \ldots \ldots \ldots \ldots
\end{aligned}
$$

The invariant ' $J{ }^{\prime 13)}$ which is independent on the coordinate transformation has close correlation with solid phase stress ${ }^{7)}$ since the phase stress is generated from the particle-to-particle collision and friction ${ }^{14)}$ related to shear mode. Therefore, special attention will be made to ' $J$ ' over a section as a function of mean flow speed $\langle U\rangle$.

\section{DEFORMATION OF SOLID PHASE IN DENSE LIQUID-SOLID FLOW IN PIPES}

\section{(1) Visual test}

Since the motion of aggregate in real fresh concrete cannot be observed, the authors utilized model concrete developed by Hashimoto et al. ${ }^{8}$. We can see aggregate by replacing mortar with the transparent viscous matrix.

For developing the compatibility of particulate flow distributed in liquid, all particles must be processed so as to quantify the strain field of dense aggregate phase. Actually, the flow of concrete in a pipe is in an axisymmetric three-dimensional condition, in which it is impossible to detect the motion of all particles in the model concrete. Then, the apparatus as shown in Fig. 5 was used to reproduce two-dimensional plane flow in straight and tapered portions made of transparent acrylic panel so as to process the motion of all particles $^{7)}$. Although two-dimensional boundary condition of experiment is different from the threedimensional actual one, it is expected to have the similar macroscopic pattern of deformation field in a section. Two-dimensional observations must be comprehensive for us to understand three-dimensional flow pattern.

\section{(2) Image data processing}

Based on the visual image data of the model concrete, the spatial averaging method of particle assembly motion was proposed based on Eulerian expression ${ }^{15)}$. The integration of velocity in Eq. (1) was numerically carried out for creating continuous velocity field as shown in Fig. 6 and followed by the strain 


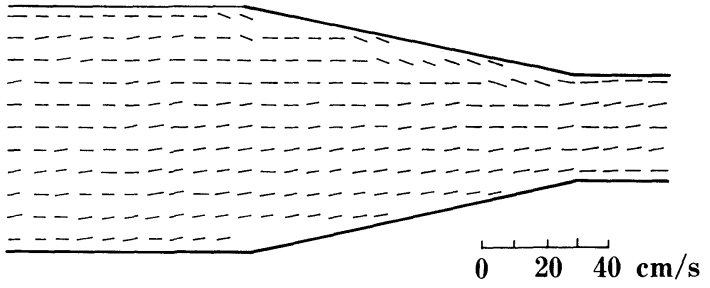

Fig. 6 Continuous velocity field generated from discrete velocity of particle assembly.

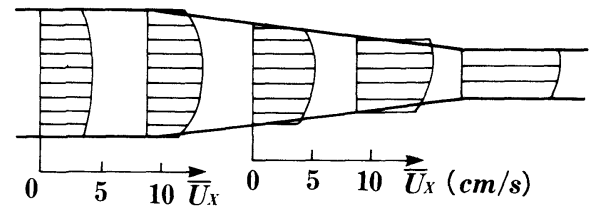

(a) Taper angle $\theta=9.5^{\circ}$

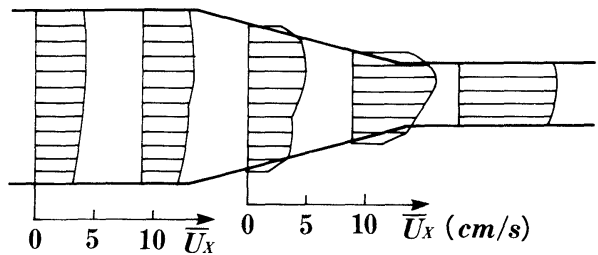

(b) Taper angle $\theta=21^{\circ}$

Fig. 7 Time averaged velocity distribution of particles

field ${ }^{15)}$. The size of the control volume $v_{c}$ was specified to be two times the maximum size of aggregate. If $v_{c}$ is less than maximum size of aggregate size or greater than several times the aggregate size, it does not represent the microscopic behavior of aggregate deformation. Therefore it is appropriate to consider two times the maximum size of aggregate as the control volume $v_{c}$.

Furthermore, flow rate, strain and its invariants in each location were averaged in time domain as

$$
\begin{aligned}
& \overline{\epsilon_{i j}}=\frac{1}{\Delta t} \int_{t}^{t+\Delta t} \epsilon_{i j} d t=\frac{1}{\Delta t} \int_{t}^{t+\Delta t} \frac{1}{2}\left(\frac{\partial u_{i}}{\partial x_{j}}+\frac{\partial u_{j}}{\partial x_{i}}\right) d t \\
& \bar{J}=\frac{1}{\Delta t} \int_{t}^{t+\Delta t} J d t \\
& \langle\bar{U}\rangle=\frac{1}{\Delta t} \int_{t}^{t+\Delta t}\langle U\rangle d t
\end{aligned}
$$

“__ indicates the time averaged value and " \langle\rangle " indicates the sectional averaged value of any parameter. The time averaging interval $\Delta t$ used in the image analysis varies from $0.65 \mathrm{sec}$. to $2.0 \mathrm{sec}$. depending on the speed of pumping.

Even though the flow would be stable, there exists some fluctuation of flow and deformation of aggregate phase in time domain. In the case of single phase liquid flow, the fluctuation of flow and associated momentum transfer expressed by Reinolds stress is prominent for liquid stress compared with the molecular shearing resistance. On the other hand, the time averaged distortion of dense solid flow described by strain rate in Eq. ( 8), (9) is considered to be greatly associated with the stress generation, compared with the momentum transfer by the flow fluctuation. Achieving the aim of this paper, firstly the authors investigate the macroscopic behavior of dense liquid-solid flow as an idealized model of concrete. The following strain measurements are based on the time averaging procedure.

a) Two-dimensional flow pattern

Time averaged velocity distribution of selected sections for tapered pipe are shown in Fig. 7, where the dense solid concentration of $35 \%$ by volume and high viscosity (' $P$ ' funnel time is $3 \mathrm{mts}$.) liquid were selected as model concrete. In this paper, "dense" liquid-solid is defined as the two-phase material with higher volume concentration of solid phase, whose contact and resulting interaction stress are not negligible. Since the volume fraction used is approximately $70 \%$ of the maximum volume fraction (i. e. $\left.G / G_{\max }=70 \%\right)$, the adopted liquid-solid is entirely "dense" two-phase material. Previous multiphase researches have been mainly oriented to slurry flow including small amount of solid particles $(<30 \%)$ and the solid plays minor role to increase momentum transfer due to turbulence and the shear rate of liquid between particles without particle-to-particle interaction effect. The velocity distribution of aggregate phase is close to plug flow in straight portions of the pipe and low tapered portions. But if the tapered angle 
is large, the plug flow no longer exists and velocity distribution of particles becomes parabolic as shown in Fig.7(b). The tapered pipes used in actual concrete pumping have very small tapered angles $(\theta \approx 1.5 \mathrm{deg}$. $)$ and high coarse aggregate concentration is utilized (approximately $30 \% \sim 45 \%$ by volume). Then, the axial flow is expected to be fairly uniform.

b) Two-dimensional deformation field denoted by strains

The distribution of time averaged strains of $\overline{\epsilon_{x x}}, \overline{\epsilon_{y y}}$, and $\overline{\epsilon_{x y}}$ for selected cross sections are shown in Fig. 8. It can be seen that in the straight portion $\overline{\epsilon_{x x}}$ and $\overline{\epsilon_{y y}}$ are very small, but that $\overline{\epsilon_{x x}}$ and $\overline{\epsilon_{y y}}$ are increasing with the mean velocity at the section as tapered cross section decreases. Here, it can be noted that $\overline{\epsilon_{x x}}$ is positive and $\overline{\epsilon_{y y}}$ is negative at any location. The shear strain $\overline{\epsilon_{x y}}$ is getting positive and negative signs and becomes zero at the center of the pipe due to symmetrical flow pattern as shown in Fig. 7 .

The sectional distribution of time averaged invariants $\bar{I}$ and $\bar{J}$ are shown in Fig. 9. The first invariant $\bar{I}$ represents the divergence rate of particles ${ }^{7)}$. Very small value of $\bar{I}$ observed over the test domain means steady state flow without segregation which the authors intended to produce. If we would use lower viscous liquid, we have unsteady flow with segregation accompanied with the increase of volume concentration in the tapered portion $^{5)}$. Since this unsteady state is not suitable for time averaging procedure, we avoid segregation process for the purpose of compatibility establishment. Then, the particle deformation in pure shear without volumetric strain rate is given by $\bar{J}$.

On the other hand, the larger shear rate intensity represented by the second invariant of $J$ is generated around the larger tapered portions and at sections with higher rate of flow. This means greater stress intensity generated due to particle-to-particle interaction with shear. The value of $\bar{J}$ has a slight non-linear distribution over a cross section with the maximum at the center of the outlet section of the pipe.

\section{(3) Sectional averaging of shear intensity}

The one-dimensional reduced compatibility will be combined with the constitutive model for particle assembly to compute sectional force $A \sigma_{s}$ derived from the aggregate interaction. Accordingly, what the reduced compatibility describes under the mean flow speed of $\langle\bar{U}\rangle$ should be necessary and sufficient for strain tensors or deformation modes which the constitutive model requires. As mentioned above, particulate flow is generally governed by the particle collision and friction mainly excited by the shear mode deformation, and experimentally obtained flow of model concrete was in pure shear where the mean strain

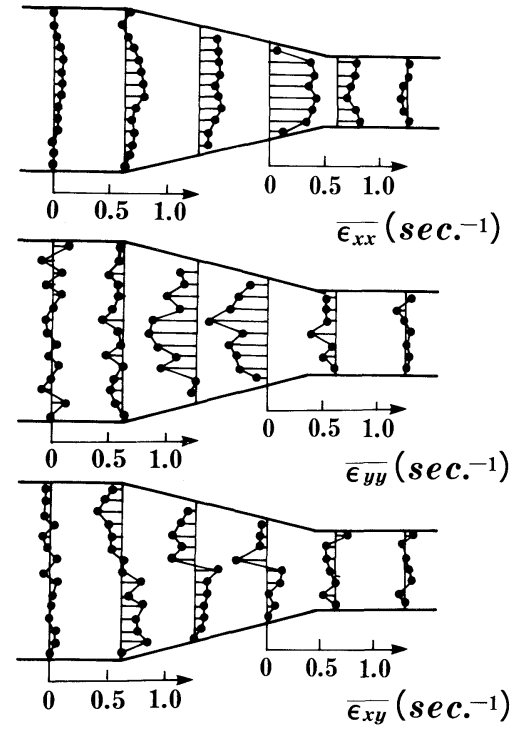

Fig. 8 Sectional distribution of time averaged strains $\overline{\epsilon_{x x}}, \overline{\epsilon_{y y}}$ and $\overline{\epsilon_{x y}}$

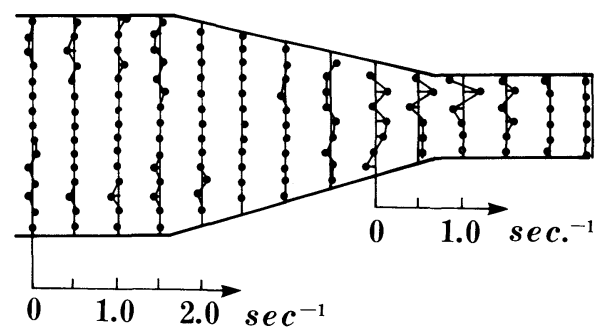

Invariant $\bar{I}$

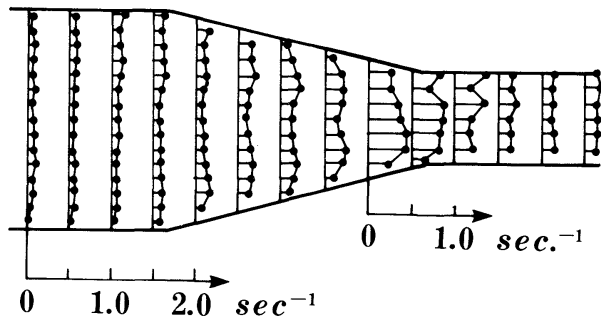

Invariant $\bar{J}$

Fig. 9 Sectional distribution of $\bar{I}$ and $\bar{J}$. 
rate $\bar{I}$ is zero. Then, the authors select the value of $\bar{J}$ as a specified value for shear intensity to be predicted by the compatibility in one-dimensional condition.

The distribution of the invariant $\bar{J}$ is described over a cross section of pipe as

$$
\begin{aligned}
& \overline{J(x, y, z)}=\langle\overline{J(x)}\rangle+J^{\prime}(x, y, z) \cdots \\
& \langle\overline{J(x)}\rangle=\frac{1}{A} \int_{A} \overline{J(x, y, z)} d y d z \cdots
\end{aligned}
$$

where $\langle\overline{J(x)}\rangle$ is the sectional averaged invariant related to particulate shear intensity, and the co-ordinate $x$ is defined paralled to the axis of pipe $\vec{S}$.

Since the sectional deviation $J^{\prime}$ is not so large compared with the mean value of $\langle\bar{J}\rangle$ in the normal tapered pipe angle, the mere sectional average $\langle\overline{J(x)}\rangle$ will be referred to in this research. If the particulate stress would be proportional to the shear rate intensity, the deviator $J^{\prime}$ might be meaningless for computing sectional mean stress $\sigma_{s}$ in Eq. (3) or force developing over a section. In case of the slow particulate shear flow, it may hold.

The typical sectional averaged $\langle\bar{J}\rangle$ is shown in Fig. 10 with sectional averaged value of $\langle\bar{I}\rangle$. We have the increasing intensity of shear mode approaching to the outlet. In the straight inlet portion, the invariant $\langle\bar{J}\rangle$ is minimum. After passing through the exit into the straight outlet pipe, shear intensity $\langle\bar{J}\rangle$ gradually decreases. This is considered to be a transient or tranquilized zone in which the momentum may be transferred by the particulate interaction. The observed flow is exactly in pure shear because of the negligibly small value of $\langle\bar{I}\rangle$ along the pipe axis.

It can be noted from Fig. 11 that the averaged $\langle\bar{J}\rangle$ is greatly reduced over the test domain for low pumping speed. Furthermore, it is understood in comparison with the results in Fig. 10 that the steeper angle of tapering excites larger shear mode intensity even though the flow speed is constant as $4.2 \mathrm{~cm} / \mathrm{s}$.

In case of the deformation around the bifurcating area of pipes, the authors experimentally clarified the effect of viscosity on the spatial distribution of $J^{7}$. However, it seems that the liquid viscosity is less effective on the distribution of $\langle\bar{J}\rangle$ as shown in Fig. 12 when the tapered angle is small $(\theta=9.5 \mathrm{deg}$. ). In the case of large tapered angle, which is not realistic indeed in actual pump transfer, there is an effect of viscosity on $\langle\bar{J}\rangle$. As shown in Fig. 13, viscosity is less effective on transition length, but increase of viscosity reduces the peak value of $\langle\bar{J}\rangle$ at the outlet of tapered pipe with taper angle of $21 \mathrm{deg}$.

\section{A PROPOSAL OF REDUCED COMPATIBILITY FOR TWO-DIMENSIONAL PIPE FLOW}

The aim of this chapter is to formulate the relation of the sectional averaged value of $\langle\bar{J}\rangle$ obtained in
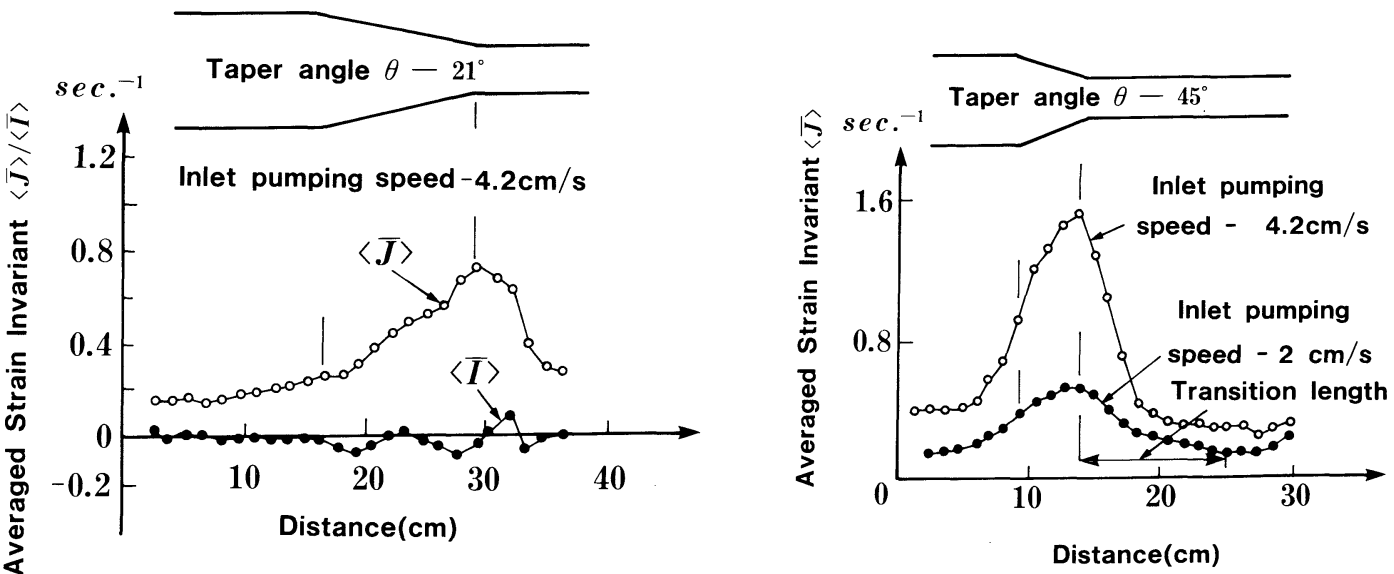

Fig. 10 Distribution of $\langle\bar{I}\rangle$ and $\langle\bar{J}\rangle$ for tapered angle $\theta=21^{\circ}$.

Fig. 11 Distribution of $\langle\bar{J}\rangle$ for tapered angle $\theta=45^{\circ}$. 


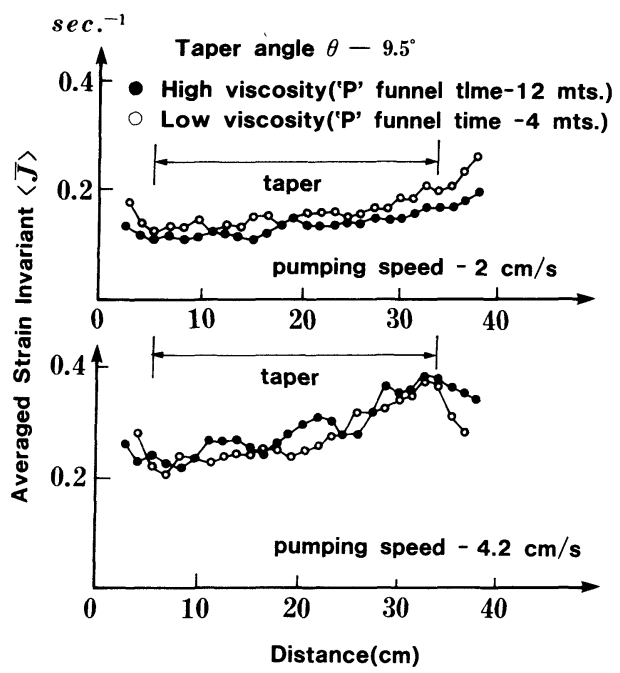

Fig. 12 Effect of liquid viscosity on $\langle\bar{J}\rangle$ for tapered angle $\theta=9.5^{\circ}$.

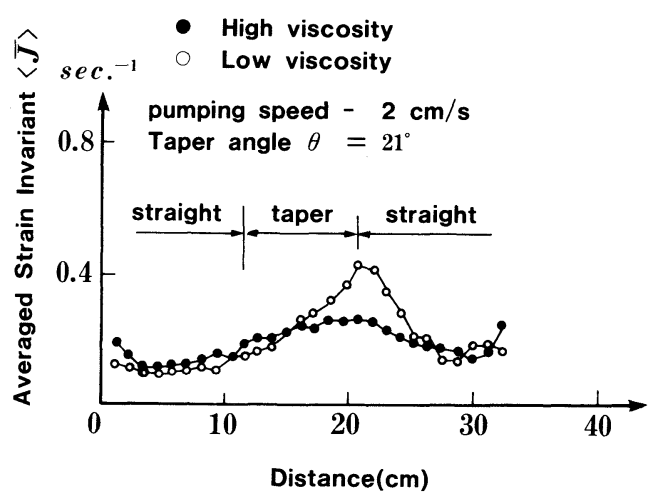

Fig. 13 Effect of liquid viscosity on $\langle\bar{J}\rangle$ for tapered angle $\theta=21^{\circ}$.

Chapter 3 versus the mean flow rate of $\langle\bar{U}\rangle$. As discussed above, the tapering angle and dimension of pipes must be included in the reduced compatibility. Exact two-dimensional compatibility gives us the following expression by substituting Eq. (6) and Eq. (9) into Eq. (12) as

$$
\begin{aligned}
& \langle\overline{J(x)}\rangle=\frac{1}{A} \int_{A} \overline{J(x, y, z)} d y d z \\
& =\frac{1}{A \Delta t} \int_{t}^{t+\Delta t} \int_{A}\left(\sqrt{\left(\frac{\epsilon_{x x}-\epsilon_{y y}}{2}\right)^{2}+\epsilon_{x y}^{2}}\right) d t d A \\
& =\frac{1}{A \Delta t} \int_{t}^{t+\Delta t} \int_{A}\left|\epsilon_{x x}-I\right| \sqrt{1+\phi^{2}} d t d A
\end{aligned}
$$

$$
\text { where } \phi=\frac{\epsilon_{x y}}{\epsilon_{x x}-I}
$$

We have a Taylor series with respect to parameter $\phi$ as

$$
\langle\overline{J(x)}\rangle=\frac{1}{A \Delta t} \int_{t}^{t+\Delta t} \int_{A}\left|\epsilon_{x x}-I\right|(\underbrace{\left.\frac{1+\frac{\phi^{2}}{2}-\frac{\phi^{4}}{8}+\frac{\phi^{6}}{16} \ldots}{2}\right)}_{\text {higher order terms }} d t d A
$$

The object of this paper is to empirically find the relation of $\langle\bar{J}\rangle$ as Eq. (15) and the mean flow $\langle\bar{U}\rangle$ as Eq. (10). Here, let us focus our attention on the first order term in Eq. (15), because the higher order terms with respect to $\phi$ may be small compared with the first linear term. In the steady state flow, there exists the exact solution of the first order compatibility in one-dimension as

$$
\begin{aligned}
& \langle\bar{J}\rangle=\left\langle\bar{J}_{1 s t}\right\rangle+J_{h}(\phi) \\
& \left\langle\bar{J}_{1 s t}\right\rangle=\frac{1}{A \Delta t} \int_{t}^{t+\Delta t} \int_{A}\left|\epsilon_{x x}-I\right| d t d A \\
& =\left|\frac{1}{A \Delta t} \int_{t}^{t+\Delta t} \int_{A}\left(\frac{\partial u_{x}}{\partial x}\right) d t d A-\frac{1}{A \Delta t} \int_{t}^{t+\Delta t} \int_{A} I d t d A\right|
\end{aligned}
$$

where $J_{h}$ is the higher order term concerned with $\phi$ in Eq. (15). The differentiation of the first term with respect to $x$ is independent on time and the second term in Eq. (17), which corresponds to $\langle\bar{I}\rangle$, can be neglected since it is very small (see Fig. 10). Then we have

$$
\left\langle J_{1 s t}\right\rangle=\left|\frac{d}{d x}\left(\frac{1}{\Delta t} \int_{t}^{t+\Delta t} \frac{1}{A}\left(\int_{A} u_{x} \cdot d A\right) \cdot d t\right)\right|=\left|\frac{d\langle\bar{U}\rangle}{d x}\right|
$$

where $\langle\bar{U}\rangle$ is the averaged flow rate in section and time domain. In steady state, we have the following 
mass-balance equation.

$\langle\overline{U(x)}\rangle \cdot A(x)=Q$ (constant. )

where $Q$ is the mass flow rate. By differentiating the right and left terms in Eq. (19) with respect to $x$ and substituting into Eq. (18), we have

$$
\left\langle\overline{J_{1 s t}}\right\rangle=\left|\frac{d A}{d x} \frac{\bar{U}\rangle}{A}\right|=\left(\frac{2}{L} \cdot \tan \theta\right)\langle\bar{U}\rangle
$$

(in case of two-dimensional duct as shown in Fig. 14)

If the higher order terms in Eq. (15) is negligible, the expression of $\left\langle\bar{J}_{1 s t}\right\rangle$ becomes the reduced compatibility which this paper aims at. Let us check the applicability of the first linear term derived from the exact solution.

As shown in Fig. 10, we have experimentally obtained shear rate intensity $\langle\bar{J}\rangle$ and the mean flow rate in each cross section. The discrepancy between the experimentally obtained $\langle\bar{J}\rangle$ and $\left\langle\bar{J}_{1 s t}\right\rangle$ obtained by Eq. (20) with measured average flow $\langle\bar{U}\rangle$ means the higher order of $J_{h}(\phi)$. Let $\beta$ denote the ratio of the higher order to the first term as

$$
\beta=\frac{\langle\bar{J}\rangle-\left\langle\bar{J}_{1 s t}\right\rangle}{\left\langle\bar{J}_{1 s t}\right\rangle}=\frac{J_{h}(\phi)}{\left\langle\bar{J}_{1 s t}\right\rangle} .
$$

The empirical relation of $\beta$ and $\left\langle\bar{J}_{1 s t}\right\rangle$ is shown in Fig. 15 which includes the experimental data of 6 flowing tests with different tapered angles $(9.5,21,45 \mathrm{deg})$ and different flow speeds $(2,4.2 \mathrm{~cm} / \mathrm{s}$ : piston speed, See Fig. 5). The higher intensity of shear rate corresponding to higher value of $\left\langle\bar{J}_{1 s t}\right\rangle$ reduces the deviation factor $\beta$ so much.

The authors reported the effect of liquid viscosity and volume concentration of aggregate on the magnitude of ' $J$ ' in a non-symmetric flow around a bifurcation ${ }^{7}$. However, in the case of this axisymmetric flow in tapered pipe, effect of liquid viscosity and the volume fraction on $\beta$ are hardly observed in Fig. 16 and Fig. 17, when a steady state of flow without segregation is produced. Therefore, we can expect $\left\langle\bar{J}_{1 s t}\right\rangle$ as one of main factors to specify the higher rate of shear intensity. Here, we have

$$
\langle\bar{J}\rangle=\left\langle\bar{J}_{1 s t}\right\rangle\left(1+\beta\left(\left\langle\bar{J}_{1 s t}\right\rangle \text {, other factors }\right)\right) \text {. }
$$

Because of the data scattering and precision of visual processor, the magnitude of higher order denoted by $\beta$ could not be formulated quantitatively when $\left\langle\bar{J}_{1 s t}\right\rangle$ is less than $0.4 \mathrm{sec}^{-1}$.

But, it is expected that the higher order term is negligible when $\left\langle\overline{J_{1 s t}}\right\rangle$ is greater than $0.4 \mathrm{sec}^{-1}$. Here, it must be noted that the evaluation of the higher order term as shown in Fig. 15 is within the dense liquid-solid flow in which the particle-to-particle interaction is prominent. The less particle interaction
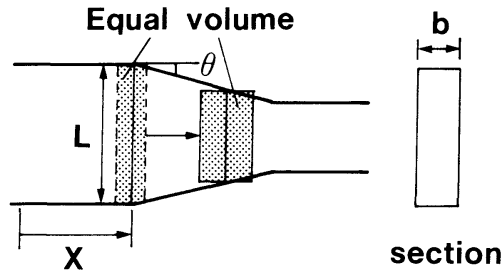

Fig. 14 Idealization of the deformation of aggregate phase.

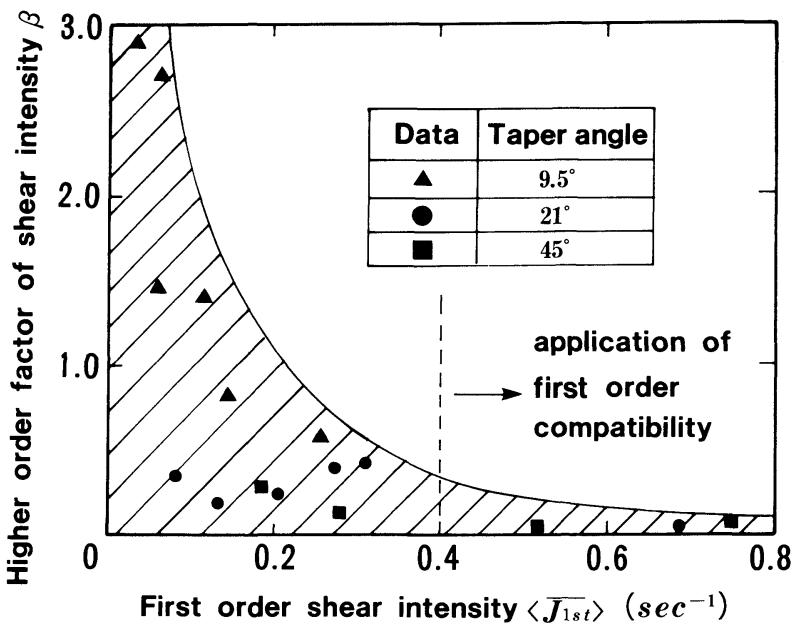

Fig. 15 Variation of $\beta$ vs. $\left\langle\overline{J_{1 s t}}\right\rangle$. 


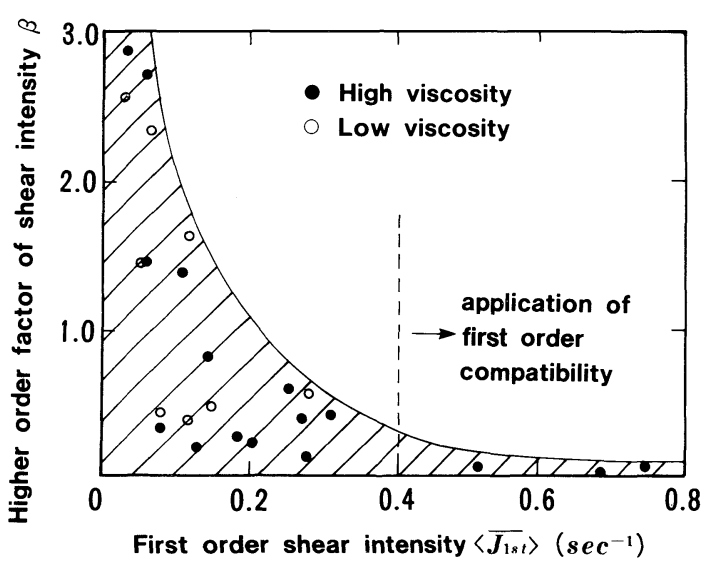

Fig. 16 Variation of $\beta$ vs. $\left\langle\overrightarrow{J_{1 s t}}\right\rangle$ for different viscosities.

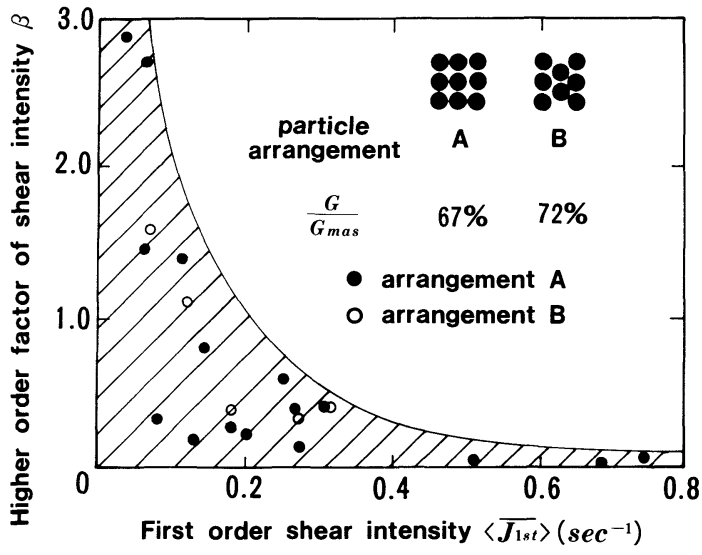

Fig. 17 Variation of $\beta$ vs. $\left.\overline{\left\langle J_{1 s t}\right.}\right\rangle$ for different particle compactions.

increases the value of $\phi$ over a cross section and greater value of $\beta$ is followed.

Let us recall the compatibility of classical beam theory where the contribution of shear strain component to the beam deflection is neglected compared with flexural action dependent on the normal strain along the beam axis. This situation is quite similar to the proposed deformational compatibility condition for tapered pipe. The omitting of the higher order terms represented by $\beta$ corresponds to the zero value of $\phi$, which means ignorance of shear strain component normal to the strain along the main flow direction. This idealized deformation corresponds to that illustrated in Fig. 14 where the distribution of flow speed is uniform.

Once the deformation of the aggregate phase is decided, the stress can be calculated depending on the stiffness of the aggregate assemblies. In actual concrete, total force acting on a pipe which can be measured, gives the sectional averaged force. There should be a way of relating sectional averaged force and sectional averaged deformation. From the experiment with actual concrete, it is possible to obtain relationship between sectional averaged force and flow rate. By combining this with proposed compatibility, it will be possible to obtain constitutive relation for deformation of aggregate assembly.

In the development of a model for aggregate stress due to deformation of aggregate phase in the tapered section, combination of the deformational compatibility model and a model for aggregate stiffness incorporating the properties of mortar can be used. By assuming the present model for deformation of aggregate phase, a model for aggregate stiffness will be evaluated experimentally in the future.

\section{EXTENSION TO THREE-DIMENSIONAL PIPE FLOW}

The compatibility in Eq. (20) and the empirically obtained higher order term as shown in Fig. 15 is applicable to two-dimensional flow in a rectangular duct. However, piping used in the civil engineering construction is in circular section in which flow of concrete is three-dimensional. Here, we can assume this flow as axisymmetric with 2 degrees of freedom concerning displacement of particles similar to the case in the experiments carried out in this paper. Then, the analogy is expected to be applied to three-dimensional compatibility for flow around tapered pipes.

The intensity of shear deformation in three-dimensional axisymmetric flow can be represented by the three-dimensional second invariant $J_{3 D}$ as

$$
\begin{aligned}
& J_{3 D}=\sqrt{\frac{\left(\epsilon_{s}-I\right)^{2}}{2}+\frac{\left(\epsilon_{r}-I\right)^{2}}{2}+\frac{\left(\epsilon_{\theta}-I\right)^{2}}{2}+\epsilon_{r s}^{2}} \\
& I=\frac{\epsilon_{s}+\epsilon_{r}+\epsilon_{\theta}}{3} \ldots \ldots \ldots \ldots \ldots \ldots \ldots \ldots \ldots \ldots \ldots \ldots \ldots \ldots \ldots \ldots \ldots \ldots \ldots \ldots
\end{aligned}
$$


where $\epsilon_{s}, \epsilon_{r}, \epsilon_{\theta}$ are axial, radial, and hoop strains.

Within a circular pipe section, we can expect isotropic deformation due to symmetry around the pipe axis as $\epsilon_{r}=\epsilon_{\theta}$. We have the similar expression as Eq. (15).

$$
\begin{aligned}
& \left\langle\overline{J_{3 D}}\right\rangle=\frac{1}{A \Delta t} \int_{t}^{t+\Delta t} \int_{A} \frac{\sqrt{3}}{2}\left|\epsilon_{s}-I\right|\left(1+\frac{\Psi^{2}}{2}-\frac{\Psi^{4}}{8}+\frac{\Psi^{6}}{16} \cdots\right) d t d A \\
& \text { higher order terms } \\
& \Psi=\frac{2 \epsilon_{r s}}{\sqrt{3}\left(\epsilon_{s}-I\right)} \\
& \left\langle\bar{J}_{1 s t, 3 D}\right\rangle=\frac{1}{A \Delta t} \int_{t}^{t+\Delta t} \int_{A} \frac{\sqrt{3}}{2}\left|\epsilon_{s}-I\right| d t d A
\end{aligned}
$$

Since the parameter $\Psi$ and the first order of sectional averaged shear intensity in three-dimension is proportional to those in two-dimension, the first order term is expected to represent the particle-toparticle interaction when we deal with dense liquid-solid with higher flow rate.

In the steady state flow with $I=0, \mathrm{Eq}$. (28) is formed similarly to Eq. (20) as

$$
\left\langle\bar{J}_{1 s t, 3 D}\right\rangle=-\frac{\sqrt{3}}{2} \frac{d A}{d x} \frac{\langle\bar{U}\rangle}{A}=\frac{\sqrt{3}}{2}\left(\frac{2}{R} \cdot \tan \theta\right)\langle\bar{U}\rangle
$$

(in case of circular pipe with radius $R$ )

The characteristic value regarding the size of section is theoretically derived as the pipe radius $R$. As the higher order term is concerned, the authors have no empirical information. However, it can be expected that we have the similar trend as shown in Fig. 15. The greater the value of $\left\langle\bar{J}_{1 s t, 30}\right\rangle$ is obtained, the less the higher order term may contribute, because the parameters $\phi$ and $\Psi$, which are related to the higher order of shear intensity, represent the same mechanical behavior of particles. These parameters, $\phi$ and $\Psi$ in two and three-dimensional compatibility correspond the profile of particle speed over a section. In case of the uniform profile compared with the axial normal strain rate as illustrated in Fig. 14, $\phi$ and $\Psi$ become zero and make the higher order negligible. As shown in Fig. 7, the profile of particle speed is relatively flatter and then the first term of sectional averaged intensity of shear is larger for dense liquid-solid flow.

Then, the first order assumption for reduced compatibility of tapering flow of dense liquid-solid material is

$$
\langle\bar{J}\rangle=\left\langle\bar{J}_{1 s t}\right\rangle=k \cdot \tan \theta \cdot\langle\bar{U}\rangle
$$

where $k=\sqrt{3} / R$ in case of circular pipe with radius $R$ and $k=2 / L$ in case of rectangular duct with depth $L$.

\section{CONCLUSION}

For dense liquid-solid flow and deformation like that in concrete, solid particle interaction due to collision, friction and rolling must be evaluated in the momentum equations for multiphase model.

Based on the so-called visualized test, a one-dimensional compatibility for deformation, which relates the mean speed of particles to the shear rate intensity of dense particle assembly denoted by the second invariant of strain rate, was proposed in tapered section. By combining the compatibility proposed in this research with the constitutive law for particle assembly, the authors expect to complete the multiphase dynamic model for fresh concrete in the future. The followings were experimentally and analytically clarified by the visualized technique for processing particles' motion using model concrete.

(1) In a two-dimensional tapered pipe flow of dense liquid-solid, the sectional averaged intensity of shear rate is dependent on the mean particle speed and tapered angle, but hardly dependent on the liquid viscosity and volume concentration of solid distributed in liquid at high concentration.

(2) The sectional averaged intensity of particles' shear rate was represented by the second invariant 
of strain rate, which was analytically integrated over a section. The linear term of shear rate intensity was experimentally found to be prominent in comparison with the higher order term when the absolute value of the first term is large.

(3) The first linear term of deformational compatibility for particle assembly is theoretically derived from the mean flow speed, tapered angle and pipe radius.

\section{ACKNOWLEDGEMENTS}

The authors are grateful to Prof. Hajime Okamura (University of Tokyo) for his valuable suggestions in connection with this work. The authors would like to acknowledge the Ministry of Education for providing financial support by Grand-in-Aid for Scientific Research No. 01850107.

\section{REFERENCES}

1) Tanabe, K. and Takase, S. : “Wishbone Tree” Pipe Layout for Concrete Pumping, IABSE Symposium, Paris-Versailles, pp. 691-692, 1987.

2) Best, J. F. and Lane, R. O. : Testing for Optimum Pumpability of Concrete, Concrete International, pp. 9-17, October 1980.

3) Wallis, G. B. : One Dimensional Two-Phase Flow, p. 182, McGraw-Hill, New York, 1969.

4) Roco, M. C. and Shook, C. A. : New Approach to Predict Concentration Distribution in Fine Particle Slurry Flows, PCH Physiochemical Hydrodynamics, Vol. 8, No.1, pp. 43-60, 1987.

5) Nanayakkara, A., Ozawa, K., Gunatilaka, D. and Maekawa, K. : Two-Phase Computational Model for Flow and Segregation of Fresh Concrete in Tapered Pipes, Proceedings of the 3 rd International Symposium on Liquid-Solid Flows, ASME, pp. 47-52, 1988.

6) Shen, H. H. and Ackermann, N. L. : Constitutive Relationships for Fluid-Solid Mixtures, J. Eng. Mech., ASCE, Vol. 108, pp. 748-763, 1982.

7) Ozawa, K., Nanayakkara, A. and Maekawa, K. : Flow and Segregation Behavior of Two-Phase Model Concrete Around Bifurcation in Pipe Lines, Proc. of JSCE, No. 408/V-11, pp. 195-203, August 1989.

8) Hashimoto, C., Maruyama, K. and Shimizu, K. : Study on Visualization of Blocking of Fresh Concrete Flowing in Pipe, Concrete Journal, JCI, No.2, pp.119-127, 1988.

9) Cundall, D. A. and Starck, O. D. L. : A Discrete Numerical Model for Granular Assemblies, Geotechnique 29, 1, pp. 47-65, 1979.

10) Tanigawa, Y., Mori, H., Watanabe, K. and Miwa, M. : Suspension Element Analysis for Flow of Fresh Concrete in Tapered Pipe, Proc. of JCI, Vol.11, No. 1, pp.727-732, July 1989.

11) Tarumi, Y. and Hakuno, M. : A Granular Assembly Simulation for the Liquefaction of Sand and Quick Sand, Bull. Earthq. Res. Inst., University of Tokyo, Vol.62, pp. 535-577, 1987.

12) Okamura, H. and Maekawa, K. : Non-Linear Finite Element Analysis of Reinforced Concrete, Proc. of JSCE, No. $360 /$ V -3. pp. 1-10, Aug. 1985.

13) Maekawa, K. and Okamura, H. : Deformational Behaviors and Constitutive Equations of Concrete Based on the Elasto-Plastic and Fracture Model, Journal of the Faculty of Engineering, The University of Tokyo (B) Vol. XXXIV, No.2. 1982.

14) Ma, D. A. and Roco, M. C. : Probabilistic Three-Dimensional Model for Slow Shearing Particulate Flow : Wet Friction, Third International Symposium on Liquid-Solid Flows, ASME, pp. 53-58, 1988.

15) Ozawa, K., Nanayakkara, A. and Maekawa, K. : Evaluation of Aggregate Particle Motion in Liquid-Solid Flows of Model Concrete, Proc. of JSCE, No. 408/V-11, pp. 187-193, August 1989. 